\title{
Clinical Feasibility of Fast 3-Dimensional Dosimetry of the Liver for Treatment Planning of Hepatocellular Carcinoma with ${ }^{90}$ Y-Microspheres
}

\author{
Arnaud Dieudonné ${ }^{1,2}$, Etienne Garin ${ }^{3}$, Sophie Laffont ${ }^{3}$, Yan Rolland ${ }^{4}$, Rachida Lebtahi ${ }^{1,2,5}$, Dominique Leguludec ${ }^{1,5}$, \\ and Isabelle Gardin 6,7 \\ ${ }^{1}$ Department of Nuclear Medicine, Beaujon Hospital, Assistance Publique-Hôpitaux de Paris (APHP), Clichy, France; ${ }^{2}$ INSERM \\ U773, Paris, France; ${ }^{3}$ Department of Nuclear Medicine, Comprehensive Cancer Institute Eugène Marquis, Rennes, France; \\ ${ }^{4}$ Department of Radiology, Comprehensive Cancer Institute Eugène Marquis, Rennes, France; ${ }^{5}$ University Paris Diderot-Paris 7 , \\ Paris, France; ${ }^{6}$ LITIS [EA 4108], University of Rouen, Rouen, France; and ${ }^{7}$ Department of Nuclear Medicine, Henri Becquerel \\ Center and $\mathrm{CHU}$, Rouen, France
}

Several treatment strategies are used for selective internal radiation therapy with ${ }^{90} \mathrm{Y}$-microspheres. The diversity of approaches does not favor the standardization of the prescribed activity calculation. To this aim, a fast 3-dimensional (3D) dosimetry method was developed for ${ }^{90} \mathrm{Y}$-microsphere treatment planning and was clinically evaluated retrospectively. Methods: Our 3D approach is based on voxel S values (VSVs) and has been implemented in the software tool VoxelDose. VSVs were previously calculated at a fine voxel size. The time-integrated activity (TIA) map is derived from pretherapeutic 99mTc-macroaggregated-albumin SPECT/CT. The fine VSV map is resampled at the voxel size of the TIA map. Then, the TIA map is convolved with the resampled VSV map to construct the 3D dose map. Data for 10 patients with 12 tumor sites treated by ${ }^{90} \mathrm{Y}$-microspheres for hepatocellular carcinoma were collected retrospectively. 3D dose maps were computed for each patient, and tumoral liver and nontumoral liver (TL and NTL, respectively) were delineated, allowing the computation of descriptive statistics (i.e., mean absorbed dose, minimum absorbed dose, and maximum absorbed dose) and dose-volume histograms. Mean absorbed doses in TL and NTL from VoxelDose were compared with those calculated with the standard partition model. Results: The estimated processing time for a complete 3D dosimetry calculation is on the order of $15 \mathrm{~min}$, including $10 \mathrm{~s}$ for the dose calculation (i.e., VSV resampling and convolution). An additional 45 min was needed for the semiautomatic and manual segmentation of TL and NTL. The mean absorbed dose $( \pm S D)$ was $422 \pm 263$ Gy for TL and 50.1 \pm 36.0 Gy for NTL. The comparison between VoxelDose and partition model shows a mean relative difference of $1.5 \%$ for TL and $4.4 \%$ for NTL. Results show a wide spread of voxel-dose values around mean absorbed dose. The minimum absorbed dose within TL ranges from 32 to $267 \mathrm{~Gy}(n=12)$. The fraction of NTL volume irradiated with at least 80 Gy ranges from $4 \%$ to $70 \%(n=10)$, and the absorbed dose from which $25 \%$ of NTL

Received Jul. 1, 2011; revision accepted Aug. 1, 2011.

For correspondance or reprints contact: Arnaud Dieudonné, Service de Médecine Nucléaire, Hôpital Beaujon, 100, Boulevard du Général Leclerc, 92110 Clichy, France.

E-mail: arnaud.dieudonne@bjn.aphp.fr

Published online Nov. 8, 2011.

COPYRIGHT @ 2011 by the Society of Nuclear Medicine, Inc. was the least irradiated ranges from 14 to $178 \mathrm{~Gy}$. Conclusion: This article demonstrates the feasibility of a fast 3D dosimetry method for ${ }^{90} \mathrm{Y}$-microspheres and highlights the potential value of a $3 \mathrm{D}$ treatment planning strategy.

Key Words: 3D-dosimetry; voxel S values; SIRT; TARE; ${ }^{90}$ Y; microspheres

J Nucl Med 2011; 52:1930-1937

DOI: 10.2967/jnumed.111.095232

O Din intrahepatic arterial administration of ${ }^{90} \mathrm{Y}$-microspheres, also called transarterial radioembolization, is a promising modality for the treatment of primary and metastatic liver cancer. The clinical efficacy and relative safety of SIRT have been demonstrated in several prospective and retrospective studies $(1,2)$. Two microsphere products are commercially available. TheraSphere (glass microspheres; MDS Nordion) and SIR-Spheres (resin microspheres; Sirtex Medical) were approved by the U.S. Food and Drug Administration in 1999 and 2002, respectively.

The efficiency of SIRT treatment is based on a vascular selectivity process responsible for a differential effect leading to a higher concentration of radioactivity within the tumor tissue than in nontumoral tissues. The more differential this effect is, the more effective the treatment should be. Selectivity of microspheres can be improved by obstructing certain vessels during a pretreatment hepatic angiogram to spare healthy tissue, but the resulting differential effect is still constrained by the arterial system. It is well known that the anatomy of the mesenteric system and the hepatic arterial bed has a high degree of variation among patients (3).

The incidence of complications after SIRT for appropriately selected patients is low (4), but serious complications have been reported when microspheres were inadvertently deposited in excessive amounts in organs other than liverthat is, extrahepatic visceral sites (5-8) and to a lesser 
extent the lungs (9). Previous studies with ${ }^{90} \mathrm{Y}$-microspheres demonstrated that the highest tolerable dose to the lungs can reach 30 Gy for a single injection (10).

Several methods have been proposed in the literature to assess the prescribed activity. Some of them are based on empiric models (11), whereas others are based on a planning treatment strategy including a dosimetry step $(12,13)$. For glass microspheres, the recommended dose to the targeted liver defined on CT images (i.e., lobe, segment, or subsegment) is between 80 and $150 \mathrm{~Gy}$, leading to a wide range of possible administered activity (14). Thus, a more restrictive range of $120 \pm 20 \mathrm{~Gy}$ is often prescribed. For resin microspheres, a first method recommends a standard amount of administered activity, which is adapted only according to the tumor size with respect to the volume of the liver. A variant of this empiric method is to adjust the administered activity also according to the body surface area (11).

The aim of treatment planning methods based on dosimetry is to optimize the tumor response and prevent complications by administering the highest possible activity to the tumor while maintaining low radiation dose to sensitive tissues such as the lungs and normal or cirrhotic liver. This method is based on the standard MIRD scheme at the organ and tissue level (15) and is called the partition model (12). This planning treatment strategy needs volume measurement of the liver and tumor tissues and the amount of implanted activity into each of these 2 compartments and lungs. This approach has been included in the standard procedure for resin microspheres (11), with a dose constraint of 80 Gy to normal liver, 70 Gy to cirrhotic liver, and no constraint to the tumor.

This diversity of approaches does not favor standardized medical practices and leads to wide variation in the prescription of the administered activity, complicating the interpretation of clinical results and metaanalysis and the treatment efficiency.

The treatment procedure outline is as follows (16). First, a pretreatment hepatic angiogram was performed, and all vessels that could transport microspheres to nontarget organs were identified and, if possible, blocked to prevent the spread of ${ }^{90} \mathrm{Y}$-microspheres outside the liver. To simulate the treatment, ${ }^{99 \mathrm{~m}} \mathrm{Tc}-$ macroaggregated-albumin $\left({ }^{99 \mathrm{~m} T c-M A A}\right.$ ) was injected into the hepatic artery in a manner similar to the application during microsphere treatment. Then, the distribution of $99 \mathrm{~m}$ Tc-MAA was visualized by $\gamma$-scintigraphy. Planar or whole-body scintigraphy was performed to identify extrahepatic accumulation of radioactivity and to assess lung shunt fraction (F). Additional SPECT and SPECT/CT scans have also been proposed to improve the sensitivity and specificity (17) in the identification of extrahepatic visceral sites at risk to select patients and prevent postinjection complications (18).

The combination of morphologic imaging (CT or CT angiography) and functional imaging (planar, whole body, SPECT, or SPECT/CT) can be used for volume and activity measurements to assess the prescribed activity according to the planning strategy used. The whole dosimetry process for the treatment planning can be time-consuming because of nondedicated software tools. Furthermore, the prescribed dose refers to a mean absorbed dose $\left(D_{\text {mean }}\right)$ without taking into account the heterogeneity of dose distribution within the liver and tumor tissue. In external-beam radiation therapy (EBRT), it has been established for many years that not only the mean dose but also the 3D dose distribution are essential parameters to predict treatment efficiency and protect organs at risk. The latter is analyzed using isodose curves and dose-volume histograms (DVHs).

Our aim was to propose a fast 3D dosimetry methodology for SIRT with ${ }^{90}$ Y-microspheres allowing the assessment of the $\mathrm{D}_{\text {mean }}$ to the tumoral liver (TL) and the nontumoral liver (NTL) isodose curves and DVH. The 3D dose calculation is based on fine voxel S values (VSVs) (19) and has been implemented in the software tool VoxelDose (20). As a proof of concept, the clinical feasibility of our 3D approach was tested retrospectively with data from 10 patients treated by ${ }^{90} \mathrm{Y}$-microspheres for hepatocellular carcinoma (HCC) at the Comprehensive Cancer Institute Eugène Marquis of Rennes (France).

\section{MATERIALS AND METHODS}

\section{D Dosimetry Principles}

The proposed 3D dosimetry approach is based on the MIRD scheme at the voxel level described in the MIRD Pamphlet 17 (21) and recently extended to any voxel dimensions (19). Briefly, the $\mathrm{D}_{\text {mean }}$ to a target voxel, $\mathrm{T}$, is the result of the contribution of itself and all surrounding voxel sources $\mathrm{S}$ :

$$
\bar{D}\left(\text { voxel }_{T}\right)=\sum_{S=0}^{N-1} \tilde{A}\left(\text { voxel }_{S}\right) \times S\left(\text { voxel }_{T} \leftarrow \text { voxel }_{S}\right), \quad \text { Eq. } 1
$$

where $\tilde{A}\left(\right.$ voxel $\left._{S}\right)$ is the time-integrated activity (TIA) within voxels $S$, and $S\left(\right.$ voxel $_{T} \leftarrow$ voxel $\left._{S}\right)$ is the absorbed dose per unit cumulated activity between each voxel.

Both can be represented as matrices. Thus, Equation 1 was implemented as a discrete convolution between the TIA map containing each individual $\tilde{A}\left(\right.$ voxel $\left._{S}\right)$ and the VSV map. The TIA map and VSV map must have the same voxel size. To the aim of calculating absorbed doses whatever the SPECT device and voxel dimension, the VSVs were previously calculated at a fine voxel size for several radionuclides and medium (i.e., soft tissues and lung) with the Monte Carlo code MCNPX (22) (Oak Ridge National Laboratory). To adapt these fine-resolution VSV sets to the TIA map voxel size, a resampling algorithm was developed (19) to create a VSV map at the desired voxel dimension. After this resampling process, the 3D dose map was calculated by convolving the TIA map with the VSV map using the fast Hartley transform. The procedure is illustrated in Figure 1.

When tissues or organs are delineated, several parameters and descriptive statistics on the 3D dose map can be computed, such as the $\mathrm{D}_{\text {mean }}$ and median, maximum, and minimum absorbed doses in the corresponding volume of interest $\left(\mathrm{D}_{\text {med }}, \mathrm{D}_{\max }\right.$, and $\mathrm{D}_{\text {min }}$ [in Gy], respectively). Several tools have also been developed for the 3D dose map analysis. Isodose curves can be drawn and the corresponding volumes calculated, as well as DVHs, from which characteristic values can be noted.

\section{Patients and Treatment with ${ }^{90} \mathrm{Y}$-Microspheres}

Data for 10 patients receiving SIRT with glass microspheres for HCC were collected to perform a retrospective dosimetry. Glass 
FIGURE 1. Diagram illustrating principle of 3D dosimetry approach. Precalculated fine VSV map is resampled at voxel size of TIA map. Then, resampled VSV map is convolved with TIA map to compute 3D absorbed dose for each individual voxel.

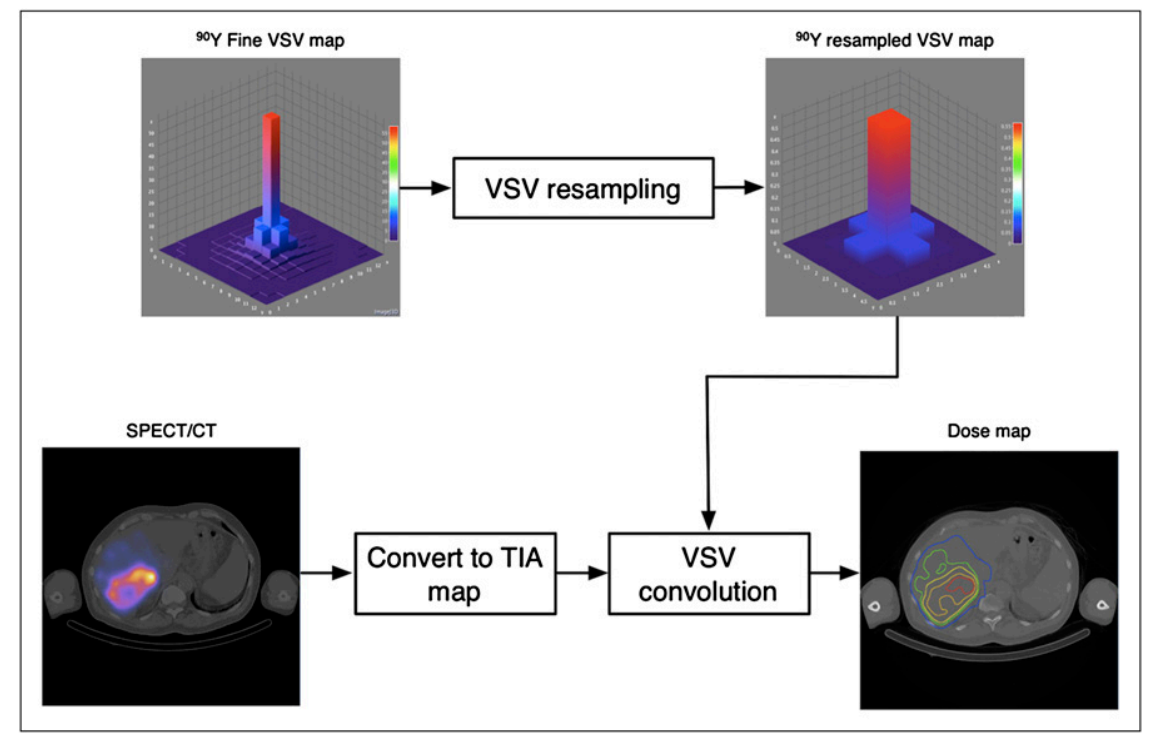

microspheres were prescribed as a first-line treatment for 6 patients and after a recurrence of HCC for 4 patients. In 5 patients, the HCC was multifocal. The average tumor diameter $( \pm \mathrm{SD})$ was $6.8 \pm 3.3 \mathrm{~cm}$.

The treatment was performed as described by Salem et al. (23) and following the instructions for use of glass microspheres (14). A pretreatment hepatic angiogram was performed to evaluate the potential lung and gastrointestinal irradiation. At the end of this first angiogram, $110 \mathrm{MBq}$ of ${ }^{99 \mathrm{~m}} \mathrm{Tc}-\mathrm{MAA}$ were injected. A planar acquisition $(256 \times 256 ; 5 \mathrm{~min})$ and SPECT/CT projections were acquired on a Symbia T2 $\gamma$-camera (Siemens Healthcare) (64 projections, $360^{\circ}, 128 \times 128,30 \mathrm{~s}$ per projection, and $130 \mathrm{kV}$ ). SPECT data were reconstructed using a 3D ordered-subset expectation maximization algorithm (5 iterations and 8 subsets) on syngo MI Workplace (Siemens Healthcare) with CT-based attenuation correction and scatter compensation based on scatter projection estimates and nonuniform collimator response compensation to recover isotropic resolution. The voxel size was $4.8 \times 4.8 \times 4.8$ $\mathrm{mm}$. CT data were reconstructed with a soft-tissue filter and a voxel size of $0.976 \times 0.976 \times 5 \mathrm{~mm}$.

The assessment of the microspheres activity to administer was based on the standard scheme proposed by the vendor (14). The activity to administer was calculated using Equation 2, with an initial objective of delivering a dose of $120 \pm 20$ Gy to the targeted liver mass, defined by the radiologist on CT data, while not exceeding a cumulative dose of $30 \mathrm{~Gy}$ to the lungs. Depending on the tumor volume, this targeted liver mass corresponds to a liver lobe, segment, or subsegment.

$$
\text { Activity to administer }(\mathrm{GBq})=\frac{\text { treated liver mass }(\mathrm{kg})}{49.67} \text {. }
$$

The value of $49.67 \mathrm{~Gy} / \mathrm{GBq}$ corresponds to the absorbed dose coefficient of ${ }^{90} \mathrm{Y}$ for a mass of $1 \mathrm{~kg}$ of soft tissue.

The percentage of $\mathrm{F}$ was estimated on planar imaging. Regions of interest, corresponding to the lungs and liver, were delineated on anterior and posterior views. The geometric mean of the number of counts on conjugate views was calculated for the liver and lungs. Then, $\mathrm{F}$ was calculated according to the following relationship:

$$
\mathrm{F}(\%)=\frac{\text { counts in lungs }}{\text { counts in lungs }+ \text { counts in liver }} \times 100 \text {. Eq. } 3
$$

The corresponding dose to lungs was calculated using Equation 4 , assuming a total mass of $1 \mathrm{~kg}$ for both lungs, as proposed by the study by Lau et al. (24).

Dose to lungs $(\mathrm{Gy})=$ activity to administer $(\mathrm{Bq}) \times \mathrm{F} / 100 \times 49.67$.

Eq. 4

The therapeutic injection was performed under angiography, in the same condition as the pretherapeutic procedure. The details of the treatment planning are summarized in Table 1.

\section{Dosimetry Assessment Using Partition Model}

For the purposes of this study, the partition model (12) was used retrospectively to estimate $D_{\text {mean }}$ to the TL and NTL, for which TL plus NTL corresponds to the total liver volume. The TL volume was determined for the reconstructed SPECT data by applying a manual threshold using volumetric analysis software on MI Workplace. The level of threshold was set up by taking into account anatomic a priori-that is, liver boundaries and tumor description from previous CT angiography scans (Table 2). The NTL volume was deduced from TL and the total liver volume. Twelve tumoral compartments, or tumoral sites, were then identified, corresponding to 2 sites for patients 5 and 6 and to 1 site for the other patients.

The absorbed dose in TL and NTL $\left(D_{T L / N T L}\right)$ was calculated according to the amount of activity $\left(A_{T L / N T L} 0\right)$ in the corresponding compartment (TL or NTL) at $\mathrm{t}=0$ (Eqs. 5 and 6).

$$
\bar{D}_{T L / N T L}(\mathrm{~Gy})=\frac{49.67 \times A_{T L / N T L_{-} 0}(\mathrm{GBq})}{m_{T L / N T L}(\mathrm{~kg})}
$$

Eq. 5

with

$$
A_{T L / N T L_{-} 0}(\mathrm{GBq})=A(\mathrm{GBq}) \times(1-\mathrm{F} / 100) \times \frac{A_{T L / N T L}}{A_{T L}+A_{N T L}} .
$$

Eq. 6 
TABLE 1

Patient Data

\begin{tabular}{ccccc}
\hline $\begin{array}{c}\text { Patient } \\
\text { no. }\end{array}$ & $\begin{array}{c}\text { Targeted } \\
\text { liver } \\
\text { mass }(\mathrm{g})\end{array}$ & $\mathrm{F}(\%)$ & $\begin{array}{c}\text { Calculated } \\
\text { activity } \\
(\mathrm{GBq})\end{array}$ & $\begin{array}{c}\text { Administered } \\
\text { activity } \\
(\mathrm{GBq})\end{array}$ \\
\hline 1 & 1,990 & 5.80 & 5 & 4.42 \\
2 & 1,191 & 18.6 & 2.5 & 2.44 \\
3 & 529 & 0.0 & 1.5 & 1.50 \\
4 & 1,781 & 3.37 & 7.5 & 7.51 \\
5 & 837 & 0.0 & 1.5 & 1.50 \\
6 & 626 & 0.0 & 1.5 & 1.32 \\
7 & 1,368 & 0.0 & 5 & 4.79 \\
8 & 961 & 0.0 & 2.5 & 2.62 \\
9 & 1,222 & 8.69 & 2.5 & 2.43 \\
10 & 886 & 2.60 & 2.5 & 2.45
\end{tabular}

Patient $(n=10)$ treatment data collected were targeted liver mass (i.e., liver lobe, segment, or subsegment defined by radiologist), F, calculated activity, and administered activity.

\section{D Dosimetry Assessment}

The following data were collected for the 10 patients: F, the administered activity, and the ${ }^{99 \mathrm{~m}}$ Tc-MAA SPECT/CT data. Additional structures were segmented to complete patient data, allowing 3D dosimetry analysis. The CT data were registered to the reconstructed SPECT data with MI Workplace, to match CT and SPECT slices without degrading in-plane CT resolution, leading to a voxel size of $0.976 \times 0.976 \times 4.8 \mathrm{~mm}$. The transformation matrix was calculated from SPECT and CT field-of-view matching. Then, the liver was manually segmented on the registered CT data with the OsiriX software tool (open source).

The TIA map for ${ }^{90} \mathrm{Y}$-microspheres was derived from the 99mTc-MAA SPECT/CT dataset, assuming similar distributions within the liver for ${ }^{90} \mathrm{Y}$-microspheres and ${ }^{99 m}$ Tc-MAA SPECT/ $\mathrm{CT}$. The $\mathrm{F}$ has been considered to determine absolute activity distribution within voxels of the liver. Then, the activity at the time of injection and the time-integrated activity for each voxel belonging to the liver were calculated. Because glass microspheres remain permanent in the liver, we considered only the physical decay of ${ }^{90} \mathrm{Y}(64.05 \mathrm{~h})$ for the time-integrated activity calculation.

The $3 \mathrm{D}$ dose maps and the descriptive statistics $-\mathrm{D}_{\text {mean }}, \mathrm{D}_{\text {med }}$, $\mathrm{D}_{\max }$, and $\mathrm{D}_{\min }$ (in Gy) - in the TL and NTL were calculated. $\mathrm{D}_{\text {mean }}$ calculated with VoxelDose was compared with $\mathrm{D}_{\text {mean }}$ calculated with the standard partition model. The percentage of relative difference between partition model and VSV approaches were calculated. DVHs were computed for TL and NTL, and the following characteristic values were extracted from DVHs in NTL: $\mathrm{V}_{80 \mathrm{~Gy}}$, corresponding to the fraction of NTL receiving at least $80 \mathrm{~Gy}$, and $\mathrm{D}_{25 \%}$, the absorbed dose for which $25 \%$ of NTL was the least irradiated.

\section{RESULTS}

Examples of 3D dose map and isodose curves are given in Figure 2 for patient 1 . The $D_{\text {mean }}$ calculated with VoxelDose was $422 \pm 263 \mathrm{~Gy}(n=12)$ for TL and $50.1 \pm 36.0 \mathrm{~Gy}(n=$ 10) for NTL. Detailed results derived from descriptive statistics are given in Tables 2 (TL) and 3 (NTL). $\mathrm{D}_{\text {mean }}$ calculated with VoxelDose in TL and NTL are in agreement with those calculated by partition model. The relative difference is less than $1.5 \%$ for TL and slightly higher for NTL (4.4\%).

Results from Tables 2 and 3 show a wide spread of voxeldose values around $\mathrm{D}_{\text {mean }}$. A more detailed distribution of absorbed dose is given in DVHs (Fig. 3 for TL and Fig. 4 for NTL). The DVHs of TL exhibit a plateau at the origin more or less extended from one patient to another. The end of the plateau corresponds to the $\mathrm{D}_{\min }$ to TL given in Table 2. Thus, for the 10 patients, the $D_{\min }$ within tumor voxels ranges from $32 \mathrm{~Gy}$ (patient 1) to $267 \mathrm{~Gy}$ (patient 7). On the other hand, the DVHs of NTL show that a high fraction of NTL was spared from high radiation doses (vertical part of the DVHs along the $y$-axes). For instance, patients 6 and 3 had at least $50 \%$ of the normal liver that received less than 2 Gy. Table 4 gives the $\mathrm{V}_{80 \text { Gy }}$ and $\mathrm{D}_{25 \%}$ for each patient.

As shown in a previous study (19), the dose calculation itself takes $10 \mathrm{~s}$ at maximum, from VSV resampling to

TABLE 2

Comparison of $D_{\text {mean }}$ to TL Calculated by Partition Model Approach and VoxelDose

\begin{tabular}{|c|c|c|c|c|c|c|c|}
\hline \multirow[b]{2}{*}{$\begin{array}{l}\text { Patient } \\
\text { no. }\end{array}$} & \multirow[b]{2}{*}{$\begin{array}{l}\text { TL mass } \\
(\mathrm{g})\end{array}$} & \multirow[b]{2}{*}{$\begin{array}{c}\text { SPECT } \\
\text { threshold } \\
\text { value (\%) }\end{array}$} & \multicolumn{4}{|c|}{$\mathrm{D}_{\text {mean }}$ to $\mathrm{TL}$} & \multirow[b]{2}{*}{$\begin{array}{l}\text { Absorbed dose } \\
\text { range in TL (Gy) }\end{array}$} \\
\hline & & & $\begin{array}{l}\text { Partition model } \\
\text { (Gy) }\end{array}$ & $\begin{array}{l}\text { VoxelDose } \\
\text { (Gy) }\end{array}$ & $\begin{array}{c}\text { VoxelDose } \\
\text { SD (Gy) }\end{array}$ & $\begin{array}{l}\text { Percentage } \\
\text { of relative } \\
\text { difference }\end{array}$ & \\
\hline 1 & 515 & 10 & $2.62 \times 10^{2}$ & $2.70 \times 10^{2}$ & $1.33 \times 10^{2}$ & 3.06 & $3.2 \times 10^{1}-8.99 \times 10^{2}$ \\
\hline 2 & 140 & 10 & $2.69 \times 10^{2}$ & $2.77 \times 10^{2}$ & $1.78 \times 10^{2}$ & 3.21 & $3.7 \times 10^{1}-1.02 \times 10^{2}$ \\
\hline 3 & 63.7 & 13 & $4.58 \times 10^{2}$ & $4.65 \times 10^{2}$ & $2.46 \times 10^{2}$ & 1.35 & $1.07 \times 10^{2}-1.42 \times 10^{3}$ \\
\hline 4 & 673 & 10 & $3.48 \times 10^{2}$ & $3.59 \times 10^{2}$ & $1.58 \times 10^{2}$ & 3.12 & $8.3 \times 10^{1}-1.66 \times 10^{3}$ \\
\hline 5 & 19.5 & 6 & $4.14 \times 10^{2}$ & $4.09 \times 10^{2}$ & $2.71 \times 10^{2}$ & -1.2 & $5.5 \times 10^{1}-1.26 \times 10^{3}$ \\
\hline \multirow[t]{2}{*}{6} & 106 & 21 & $2.50 \times 10^{2}$ & $2.54 \times 10^{2}$ & $1.32 \times 10^{2}$ & 1.41 & $4.0 \times 10^{1}-7.45 \times 10^{2}$ \\
\hline & 61.1 & & $2.42 \times 10^{2}$ & $2.44 \times 10^{2}$ & $1.20 \times 10^{2}$ & 0.85 & $6.6 \times 10^{1}-6.93 \times 10^{2}$ \\
\hline \multirow[t]{2}{*}{7} & 25.3 & 6 & $1.18 \times 10^{3}$ & $1.19 \times 10^{3}$ & $4.54 \times 10^{2}$ & 0.45 & $2.67 \times 10^{2}-2.38 \times 10^{3}$ \\
\hline & 55.1 & & $3.48 \times 10^{2}$ & $3.49 \times 10^{2}$ & $9.89 \times 10^{2}$ & 0.44 & $9.7 \times 10^{1}-6.55 \times 10^{2}$ \\
\hline 8 & 99.7 & 7 & $6.14 \times 10^{2}$ & $6.21 \times 10^{2}$ & $6.22 \times 10^{2}$ & 1.20 & $9.5 \times 10^{1}-4.08 \times 10^{2}$ \\
\hline 9 & 118 & 10 & $3.18 \times 10^{2}$ & $3.26 \times 10^{2}$ & $1.83 \times 10^{2}$ & 2.34 & $4.1 \times 10^{1}-1.22 \times 10^{2}$ \\
\hline 10 & 222 & 4 & $3.01 \times 10^{2}$ & $3.07 \times 10^{2}$ & $2.30 \times 10^{2}$ & 2.09 & $5.8 \times 10^{1}-2.22 \times 10^{2}$ \\
\hline Mean $\pm S D$ & $175 \pm 206$ & & $4.17 \times 10^{2} \pm 2.63 \times 10^{2}$ & $4.16 \times 10^{2} \pm 2.63 \times 10^{2}$ & & $1.20 \pm 1.32$ & \\
\hline
\end{tabular}



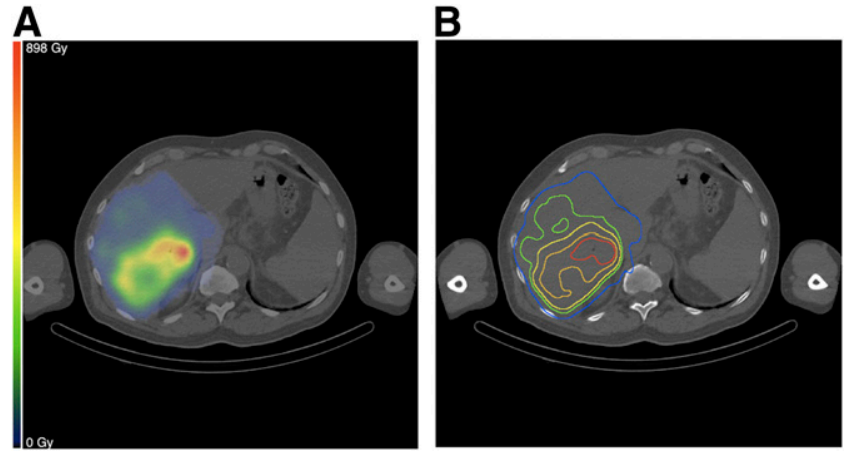

FIGURE 2. 3D dosimetry results for patient 1. (A) Transverse slice of 3D dose map fused with CT. (B) Isodose curves superimposed on transverse slice of CT. Blue curve $=$ isodose $1 \%$ of maximum dose; green curve $=$ isodose $5 \%$ of maximum dose; yellow curve $=$ isodose $10 \%$ of maximum dose; orange curve = isodose $30 \%$ of maximum dose; red curve $=$ isodose $50 \%$ of maximum dose.

convolution. The estimated processing time for a complete 3D dosimetry calculation is $15 \mathrm{~min}$, including SPECT quantitation, SPECT/CT registration, and TL definition from SPECT thresholding. Additional manual delineation of the liver increased the whole processing time to $1 \mathrm{~h}$. This delineation is a prerequisite to assessing descriptive statistics and DVH for NTL.

\section{DISCUSSION}

The aim of this work was to propose a fast 3D dosimetry methodology for ${ }^{90}$ Y-SIRT allowing the assessment of the $\mathrm{D}_{\text {mean }}$ to the targeted liver and the nontargeted liver, dose distribution heterogeneities, DVH, and, thus, the prescribed dose and activity that can be administered. The dose calculation itself is fast $(<10 \mathrm{~s})$. To obtain this speed, VSVs were previously calculated at a fine voxel size for several radionuclides and media (i.e., soft tissues, lung) using Monte Carlo simulations (19). To adapt these fine-resolution VSV sets to the $\gamma$-camera voxel dimension, a resampling algorithm was developed to create a VSV map at the desired voxel size. Another advantage is that the computation time is not related to the heterogeneity of the activity distribution within the sources, as is the case with Monte Carlo approaches. The estimated processing time for a complete 3D dosimetry calculation is $15 \mathrm{~min}$, including SPECT quantitation, SPECT/CT registration, and TL definition from SPECT thresholding. At the end of the process, the 3D dose map can be visualized, and isodose curves can be drawn. If one wants to assess descriptive statistics and DVH on NTL, additional liver delineation must be performed, with an additional time of $45 \mathrm{~min}$ with voxels of $0.976 \times 0.976 \times 4.8 \mathrm{~mm}$. To increase the speed and improve the reproducibility of processing it would be interesting to develop an automatic delineation algorithm of the liver on CT data. A 3D dosimetry approach based on dose kernel convolution (25) has been previously proposed in the literature for ${ }^{90} \mathrm{Y}$-microspheres and was evaluated on the data of 1 patient. Our approach has been developed to be used for any voxel dimension and validated with comparison to direct Monte Carlo simulations (19). This study demonstrates the feasibility of its application in clinical routine. The main drawback of our approach is that it does not take into account tissue heterogeneities. In the case of ${ }^{90} \mathrm{Y}$, which is a pure $\beta$-emitter with a limited penetration in soft tissue (mean, $2.5 \mathrm{~mm}$; maximum, $11 \mathrm{~mm}$ ), the impact of tissue heterogeneities on dose computation would remain negligible when working inside the liver. Dose perturbations at the liver boundaries cannot be simulated by our approach. However, considering previous work by Buffa and Verhaegen on interface dosimetry (26), this shortcoming would have a limited influence on the DVH analysis.

Because of the limited tissue penetration of ${ }^{90} \mathrm{Y}$, Gulec et al. (27) have performed a hepatic structural dosimetry using a Monte Carlo modeling approach based on lobular microanatomy. These results are in agreement with a previous 3D in vitro dosimetry study using hepatic arterial infusion of ${ }^{90} \mathrm{Y}$-microspheres for a VX2 tumor model in New Zealand White rabbits (24). The nonuniformity of

TABLE 3

Comparison of $D_{\text {mean }}$ to NTL Calculated by Partition Model Approach and VoxelDose

\begin{tabular}{|c|c|c|c|c|c|c|}
\hline \multirow[b]{2}{*}{$\begin{array}{l}\text { Patient } \\
\text { no. }\end{array}$} & \multirow[b]{2}{*}{$\begin{array}{l}\text { NTL mass } \\
(\mathrm{g})\end{array}$} & \multicolumn{4}{|c|}{$\mathrm{D}_{\text {mean }}$ to $\mathrm{NTL}$} & \multirow[b]{2}{*}{$\begin{array}{c}\text { Absorbed dose range } \\
\text { in NTL (Gy) }\end{array}$} \\
\hline & & $\begin{array}{c}\text { Partition } \\
\text { model (Gy) }\end{array}$ & $\begin{array}{l}\text { VoxelDose } \\
\text { (Gy) }\end{array}$ & $\begin{array}{l}\text { VoxelDose } \\
\text { SD (Gy) }\end{array}$ & $\begin{array}{l}\text { Percentage of } \\
\text { relative difference }\end{array}$ & \\
\hline 1 & 1,605 & $2.94 \times 10^{1}$ & $2.94 \times 10^{1}$ & $2.49 \times 10^{1}$ & 0.02 & $0-1.90 \times 10^{2}$ \\
\hline 2 & 1,151 & $4.38 \times 10^{1}$ & $4.53 \times 10^{1}$ & $3.10 \times 10^{1}$ & 3.46 & $0-2.42 \times 10^{2}$ \\
\hline 3 & 1,444 & $2.78 \times 10^{1}$ & $2.90 \times 10^{1}$ & $5.63 \times 10^{1}$ & 4.27 & $0-4.27 \times 10^{2}$ \\
\hline 4 & 2,371 & $4.61 \times 10^{1}$ & $4.85 \times 10^{1}$ & $4.81 \times 10^{1}$ & 5.16 & $0-4.03 \times 10^{2}$ \\
\hline 5 & 1,115 & $5.55 \times 10^{1}$ & $5.72 \times 10^{1}$ & $6.22 \times 10^{1}$ & 3.04 & $0-5.19 \times 10^{2}$ \\
\hline 6 & 1,532 & $1.25 \times 10^{1}$ & $1.37 \times 10^{1}$ & $2.7 \times 10^{1}$ & 8.90 & $0-2.72 \times 10^{2}$ \\
\hline 7 & 1,211 & $1.38 \times 10^{2}$ & $1.44 \times 10^{2}$ & $1.37 \times 10^{2}$ & 4.16 & $0-2.26 \times 10^{3}$ \\
\hline 8 & 1,199 & $4.85 \times 10^{1}$ & $5.11 \times 10^{1}$ & $7.19 \times 10^{1}$ & 5.52 & $0-6.91 \times 10^{2}$ \\
\hline 9 & 1,121 & $5.47 \times 10^{1}$ & $5.69 \times 10^{1}$ & $6.46 \times 10^{1}$ & 4.11 & $0-4.91 \times 10^{2}$ \\
\hline 10 & 1,626 & $2.50 \times 10^{1}$ & $2.63 \times 10^{1}$ & $4.47 \times 10^{1}$ & 5.18 & $0-7.75 \times 10^{2}$ \\
\hline Mean $\pm S D$ & $1,503 \pm 572$ & $4.81 \times 10^{1} \pm 3.45 \times 10^{1}$ & $5.01 \times 10^{1} \pm 3.60 \times 10^{1}$ & & $4.38 \pm 2.23$ & \\
\hline
\end{tabular}




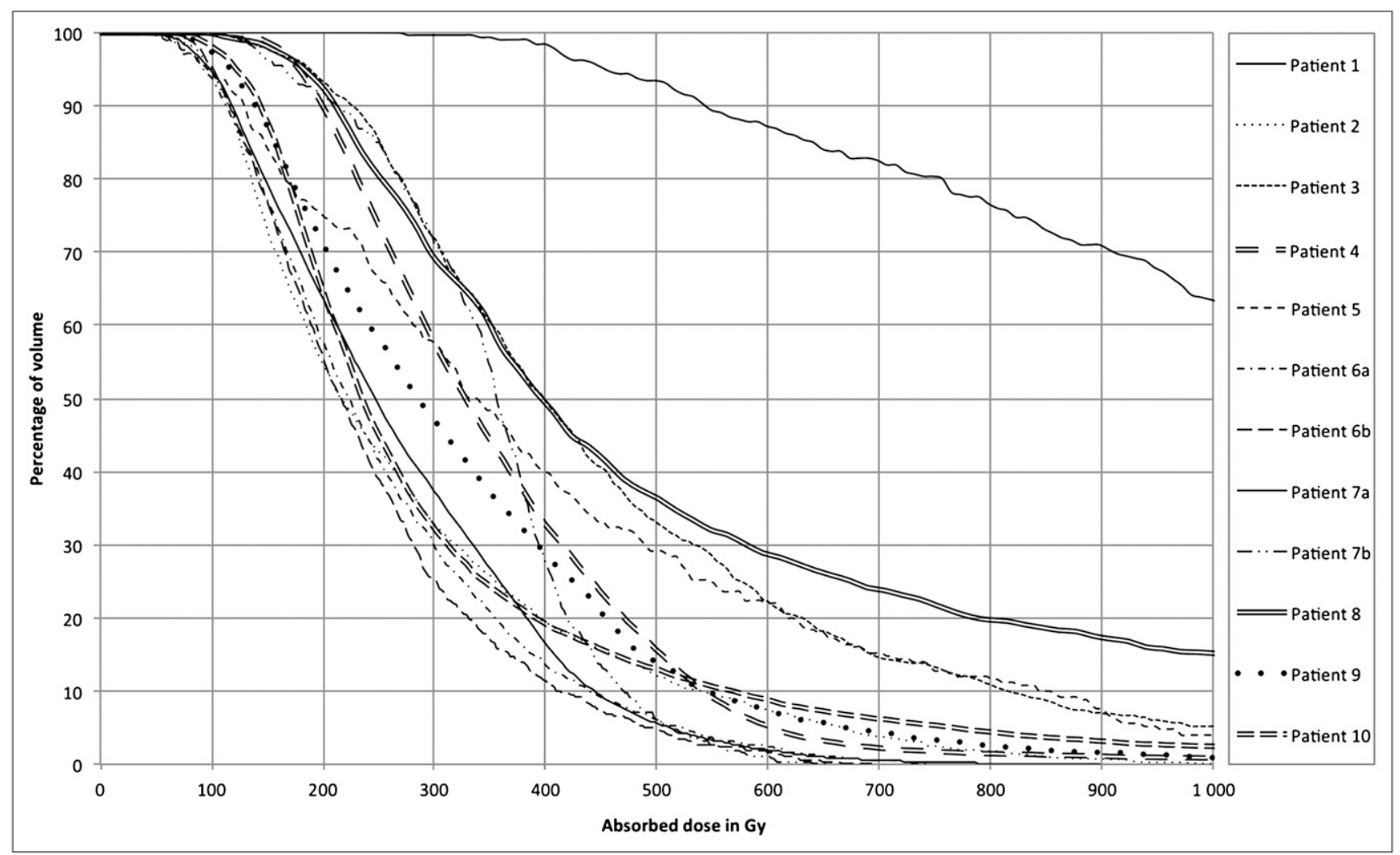

FIGURE 3. DVH in TL for each patient. Patients 6 and 7 had 2 tumor sites.

dose deposition in TL and NTL was shown on liver tissue samples (200 $\mu \mathrm{m}$ thick). This cellular dosimetry gave some interesting complementary information on dose distribution heterogeneity at a microanatomy level.

Our approach is based on dosimetry computation at the voxel level. Although this highlights a wide range of dose heterogeneity, one can argue that the lack of reliable activity measurement at the voxel level mainly is due to the limited spatial resolution of $\gamma$-camera, leading to a high partial-volume effect for structures below 2-3 times the full width at half maximum of the impulse response. Even if we keep in mind that 3D dosimetry from SPECT or PET quantitation suffers from potential partial-volume effect and cannot reveal heterogeneities at a submillimeter level, 3D dosimetry is a promising modality for a better control of differential effect and the establishment of a dose-effect relationship.

The use of ${ }^{99 \mathrm{~m} T c-M A A ~ S P E C T / C T ~ d a t a ~ f o r ~}{ }^{90}$ Y-microsphere treatment planning is conditioned by the similarity between ${ }^{99 \mathrm{~m}} \mathrm{Tc}-\mathrm{MAA}$ and ${ }^{90} \mathrm{Y}$-microsphere liver perfusion. Indeed, both injections should be done under the same conditions-that is, catheter placement and injection technique. Posttherapeutic ${ }^{90}$ Y-bremsstrahlung SPECT/CT or ${ }^{90}$ Y-PET/ CT (28) could be used as a validation for pretherapeutic dosimetry with an objective measurement of the similarity (29).

The clinical feasibility of our 3D approach was tested retrospectively on the data of 10 patients treated by SIRT with ${ }^{90}$ Y-microspheres for HCC. $\mathrm{D}_{\text {mean }}$ calculated with VoxelDose in TL and NTL are in good agreement with those calculated by the partition model (Tables 2 and 3). The $\mathrm{D}_{\text {mean }}$ calculated by VoxelDose is slightly higher than that calculated by the partition model. This result may be partly explained by the fact that in the partition model, an absorbed dose coefficient of $49.67 \mathrm{~Gy} / \mathrm{GBq}$ for $1 \mathrm{~kg}$ of tissue for ${ }^{90} \mathrm{Y}$ is taken, assuming an absorbed fraction of 1 for every compartment. This constant value does not take into account the cross-dose contribution as a first approximation due to the limited penetration of ${ }^{90} \mathrm{Y}$ emissions.

The results from Tables 2 and 3 show a significant dispersion of voxel-dose values around the $\mathrm{D}_{\text {mean }}$ in TL and NTL. DVHs (Figs. 3 and 4), as well as Table 4, give a more detailed view of this dispersion. The shape of DVHs is in agreement with those found by Sarfaraz et al. (25) for 1 patient. These DVHs could have a potential value in dose prescription as in EBRT. For the TL, one of the main interesting parameters is likely $D_{\min }$ (Table 2) to ensure that the entire TL receives at least a fixed minimal dose, $D_{\min }$ playing the role of $\mathrm{D}_{\mathrm{EBRT}}$, the prescribed dose in EBRT. For the NTL, a constraint based on a $D_{\text {mean }}$ is probably not the most appropriate planning treatment strategy (25) because of the difference of dose distribution among patients. For instance, patients 1 and 6 received at least 80 Gy to the same fraction of NTL volume (Table $4, \mathrm{~V}_{80}$ Gy $=4 \%$ ), 


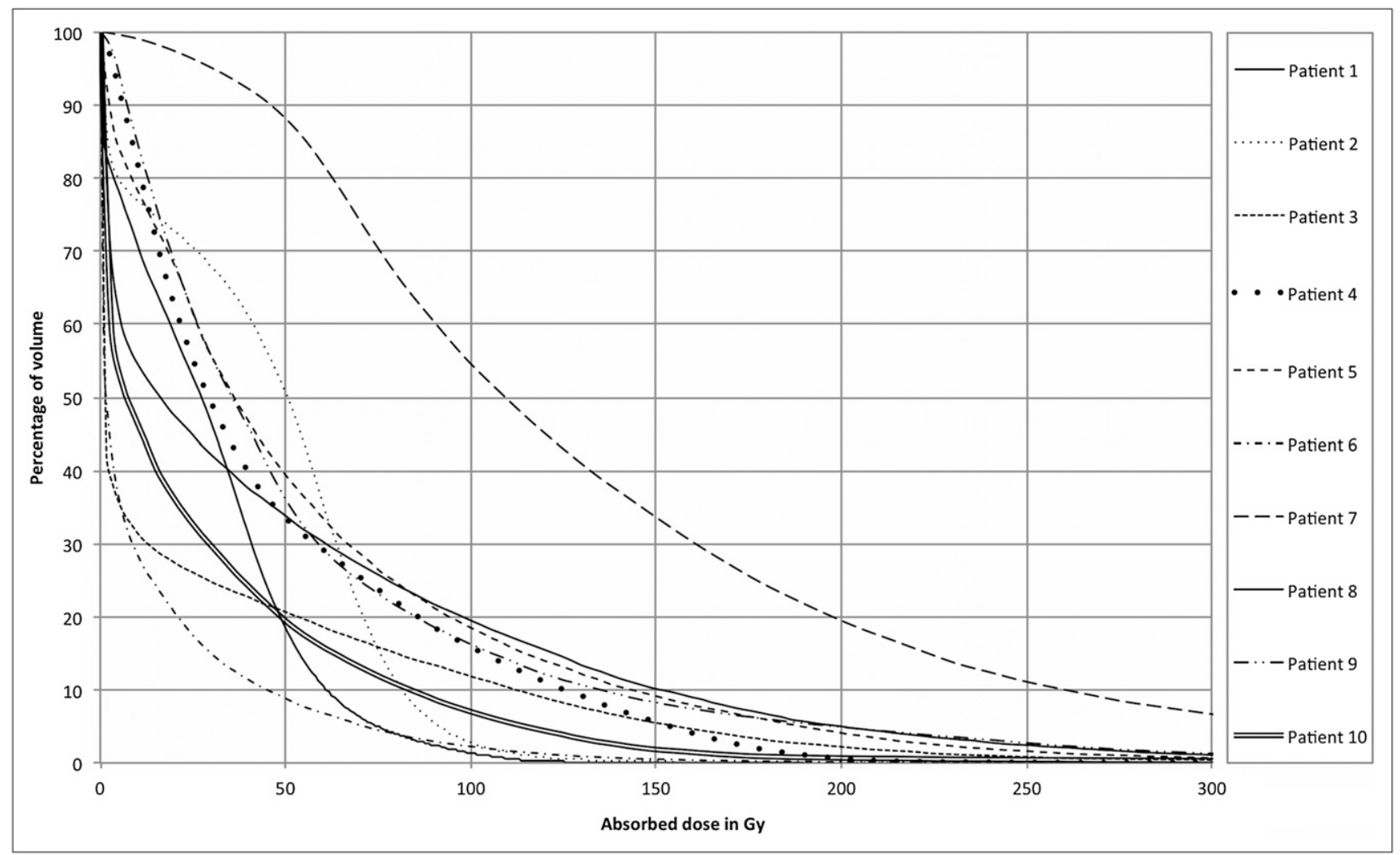

FIGURE 4. DVH in NTL for each patient.

whereas $75 \%$ of the NTL received less than 43 Gy (patient 1) and 14 Gy (patient 6) corresponding to $D_{25 \%}$. A strategy based on preserving a fraction $V_{\text {Dmin }}$ (volume irradiated by a minimum dose $\mathrm{D}_{\min }$ ) of NTL should be more appropriate to take into account the residual hepatic functional reserve than $\mathrm{D}_{\text {mean }}$. For instance, in EBRT, Lee et al. (30) have proposed dose prescription guidelines based on the fraction $\mathrm{V}_{50 \%}$ (volume irradiated by $50 \%$ of the isocenter dose) of normal liver treated. The fraction $\mathrm{V}_{50 \%}$ is divided into the following 4 intervals: if $\mathrm{V}_{50 \%}$ is less than $25 \%$, then $\mathrm{D}_{\mathrm{EBRT}}$ equals $59.4 \mathrm{~Gy}$; if $\mathrm{V}_{50 \%}$ is between $25 \%$ and $49 \%$, then $\mathrm{D}_{\text {EBRT }}$ is between 45 and $54 \mathrm{~Gy}$; if $\mathrm{V}_{50 \%}$ is between $50 \%$ and $75 \%$, then $\mathrm{D}_{\mathrm{EBRT}}$ is between 30.6 and $41.4 \mathrm{~Gy}$; and if

TABLE 4

Patient $\mathrm{V}_{80}$ Gy and $\mathrm{D}_{25 \%}$ in NTL

\begin{tabular}{ccc}
\hline Patient no. & $\mathrm{V}_{80}$ Gy $(\%)$ & $D_{25 \%}(\mathrm{~Gy})$ \\
\hline 1 & 4 & 43 \\
2 & 11 & 67 \\
3 & 15 & 28 \\
4 & 22 & 71 \\
5 & 25 & 79 \\
6 & 4 & 14 \\
7 & 70 & 178 \\
8 & 24 & 78 \\
9 & 21 & 69 \\
10 & 11 & 40 \\
\hline
\end{tabular}

$\mathrm{V}_{50 \%}$ is greater than $75 \%$, no treatment is administered. Even if there is not yet a consensus regarding which level radiation dose can be safely delivered to normal and pathologic liver, either in EBRT (31) or in SIRT, the use of a $3 \mathrm{D}$ planning treatment strategy should have a potential value in SIRT.

\section{CONCLUSION}

To improve the treatment efficiency of SIRT by ${ }^{90} \mathrm{Y}$ microspheres, a fast 3D dosimetry methodology was developed allowing the definition of the amount of activity to administer. The clinical feasibility of our 3D approach was tested retrospectively on the data for 10 patients treated by SIRT with ${ }^{90}$ Y-microspheres for HCC, leading to a highlighting of the potential value of a $3 \mathrm{D}$ planning treatment strategy in SIRT.

\section{DISCLOSURE STATEMENT}

The costs of publication of this article were defrayed in part by the payment of page charges. Therefore, and solely to indicate this fact, this article is hereby marked "advertisement" in accordance with 18 USC section 1734.

\section{ACKNOWLEDGMENT}

No potential conflict of interest relevant to this article was reported. 


\section{REFERENCES}

1. Salem R, Thurston KG. Radioembolization with 90Yttrium microspheres: a state-of-the-art brachytherapy treatment for primary and secondary liver malignancies: part 1 -technical and methodologic considerations. $J$ Vasc Interv Radiol. 2006;17:1251-1278.

2. Gulec SA, Siegel JA. Posttherapy radiation safety considerations in radiomicrosphere treatment with ${ }^{90}$ Y-microspheres. J Nucl Med. 2007;48:2080-2086.

3. Ahmadzadehfar H, Biersack H, Ezziddin S. Radioembolization of liver tumors with yttrium-90 microspheres. Semin Nucl Med. 2010;40:105-121.

4. Abdalla EK, Vauthey J, Ellis LM, et al. Recurrence and outcomes following hepatic resection, radiofrequency ablation, and combined resection/ablation for colorectal liver metastases. Ann Surg. 2004;239:818-825, discussion 825-827.

5. Dawson LA, Normolle D, Balter JM, McGinn CJ, Lawrence TS, Haken Ten RK. Analysis of radiation-induced liver disease using the Lyman NTCP model. Int J Radiat Oncol Biol Phys. 2002;53:810-821.

6. Naymagon S, Warner RRP, Patel K, et al. Gastroduodenal ulceration associated with radioembolization for the treatment of hepatic tumors: an institutional experience and review of the literature. Dig Dis Sci. 2010;55:2450-2458.

7. Aloia TA, Barakat $\mathrm{O}$, Connelly J, et al. Gastric radiation enteritis after intraarterial yttrium-90 microsphere therapy for early stage hepatocellular carcinoma. Exp Clin Transplant. 2009;7:141-144.

8. Murthy R, Brown DB, Salem R, et al. Gastrointestinal complications associated with hepatic arterial Yttrium-90 microsphere therapy. J Vasc Interv Radiol. 2007;18:553-561.

9. Salem R, Parikh P, Atassi B, et al. Incidence of radiation pneumonitis after hepatic intra-arterial radiotherapy with yttrium- 90 microspheres assuming uniform lung distribution. Am J Clin Oncol. 2008;31:431-438.

10. Leung TW, Lau WY, Ho SK, et al. Radiation pneumonitis after selective internal radiation treatment with intraarterial 90yttrium-microspheres for inoperable hepatic tumors. Int J Radiat Oncol Biol Phys. 1995;33:919-924.

11. Sirtex Medical. Sirtex Medical Training Manual: Training Program Physicians and Institutions. Lane Cove, New South Wales, Australia: Sirtex Medical.

12. Ho S, Lau WY, Leung TW, et al. Partition model for estimating radiation doses from yttrium-90 microspheres in treating hepatic tumours. Eur J Nucl Med. 1996;23:947-952.

13. Gulec SA, Mesoloras G, Stabin M. Dosimetric techniques in ${ }^{90}$ Y-microsphere therapy of liver cancer: the MIRD equations for dose calculations. J Nucl Med. 2006;47:1209-1211.

14. TheraSphere ${ }^{\circledR}$ Yttrium-90 Glass Microspheres [package insert]. 2005. Available at: http://www.mdsnordion.com/. Accessed November 3, 2011.

15. Loevinger R, Budinger TF, Watson EE. MIRD primer for absorbed dose calculations. Revised. New York, NY: The Society of Nuclear Medicine; 1991.

16. Kennedy A, Nag S, Salem R, et al. Recommendations for radioembolization of hepatic malignancies using yttrium-90 microsphere brachytherapy: a consensus panel report from the Radioembolization Brachytherapy Oncology Consortium. Int J Radiat Oncol Biol Phys. 2007;68:13-23.
17. Hamami ME, Poeppel TD, Muller S, et al. SPECT/CT with ${ }^{99 m}$ Tc-MAA in radioembolization with ${ }^{90} \mathrm{Y}$ microspheres in patients with hepatocellular cancer. J Nucl Med. 2009;50:688-692.

18. Ahmadzadehfar H, Sabet A, Biermann K, et al. The significance of $99 \mathrm{~m}$ Tc-MAA SPECT/CT liver perfusion imaging in treatment planning for ${ }^{90} \mathrm{Y}$-microsphere selective internal radiation treatment. J Nucl Med. 2010;51:1206-1212.

19. Dieudonné A, Hobbs RF, Bolch WE, Sgouros G, Gardin I. Fine-resolution voxel $\mathrm{S}$ values for constructing absorbed dose distributions at variable voxel size. J Nucl Med. 2010;51:1600-1607.

20. Gardin I, Bouchet LG, Assié K, et al. Voxeldose: a computer program for 3-D dose calculation in therapeutic nuclear medicine. Cancer Biother Radiopharm. 2003;18:109-115.

21. Bolch WE, Bouchet LG, Robertson JS, et al. MIRD pamphlet No. 17: the dosimetry of nonuniform activity distributions-radionuclide $\mathrm{S}$ values at the voxel level. Medical Internal Radiation Dose Committee. J Nucl Med. 1999;40 (suppl):11S-36S.

22. Hendricks JS, McKinney GW, Waters SW. MCNPX Extended Version 2.5.0. LAUR-05-2675. Los Alamos, NM: Los Alamos National Laboratory; 2005.

23. Salem R, Thurston KG, Carr BI, Goin JE, Geschwind JH. Yttrium-90 microspheres: radiation therapy for unresectable liver cancer. $J$ Vasc Interv Radiol. 2002; 13:S223-S229.

24. Lau WY, Ho S, Leung TW, et al. Selective internal radiation therapy for nonresectable hepatocellular carcinoma with intraarterial infusion of 90yttrium microspheres. Int J Radiat Oncol Biol Phys. 1998;40:583-592.

25. Sarfaraz M, Kennedy AS, Lodge MA, Li XA, Wu X, Yu CX. Radiation absorbed dose distribution in a patient treated with yttrium-90 microspheres for hepatocellular carcinoma. Med Phys. 2004;31:2449-2453.

26. Buffa FM, Verhaegen F. Backscatter and dose perturbations for low- to mediumenergy electron point sources at the interface between materials with different atomic numbers. Radiat Res. 2004;162:693-701.

27. Gulec S, Sztejnberg M, Siegel J, Jevremovic T, Stabin MG. Hepatic structural dosimetry in ${ }^{90} \mathrm{Y}$ microsphere treatment: A Monte Carlo modeling approach based on lobular microanatomy. J Nucl Med. 2010;51:301-310.

28. Gates V, Esmail A, Marshall K, Spies S, Salem R. Internal pair production of ${ }^{90} \mathrm{Y}$ permits hepatic localization of microspheres using routine PET: proof of concept. J Nucl Med. 2011;52:72-76.

29. Knesaurek K, Machac J, Muzinic M, DaCosta M, Zhang Z, Heiba S. Quantitative comparison of yttrium-90 $\left({ }^{90} \mathrm{Y}\right)$-microspheres and technetium- $99 \mathrm{~m}\left({ }^{99 \mathrm{~m}} \mathrm{Tc}\right)$ macroaggregated albumin SPECT images for planning ${ }^{90} \mathrm{Y}$ therapy of liver cancer. Technol Cancer Res Treat. 2010;9:253-262.

30. Lee IJ, Seong J, Shim SJ, Han KH. Radiotherapeutic parameters predictive of liver complications induced by liver tumor radiotherapy. Int J Radiat Oncol Biol Phys. 2009;73:154-158.

31. Seong J. Challenge and hope in radiotherapy of hepatocellular carcinoma. Yonsei Med J. 2009;50:601-612. 